Supplement of

\title{
Modeling organic aerosol over Europe in summer conditions with the VBS-GECKO parameterization: sensitivity to secondary organic compound properties and IVOC (intermediate-volatility organic compound) emissions
}

Victor Lannuque et al.

Correspondence to: Victor Lannuque (victor.lannuque@lisa.u-pec.fr) and Florian Couvidat (florian.couvidat@ineris.fr)

The copyright of individual parts of the supplement might differ from the CC BY 4.0 License. 


\section{Table S1 - The $\mathrm{H}^{2} \mathrm{O}$ mechanism}

\begin{tabular}{|c|c|}
\hline Reactions & $\begin{array}{l}\text { Kinetic rate parameters } \\
\left(\mathrm{s}^{-1} \text { or molecule }{ }^{-1} \cdot \mathrm{cm}^{3} \cdot \mathrm{s}^{-1}\right)\end{array}$ \\
\hline $\mathrm{ISOP}+\mathrm{OH} \rightarrow \mathrm{ISOR}+\mathrm{OH}$ & $2.54 \times 10^{-11} \times \exp (408 / \mathrm{T})$ \\
\hline $\mathrm{ISOP}+\mathrm{NO} 3 \rightarrow \mathrm{ISON}+\mathrm{NO} 3$ & $3.03 \times 10^{-12} \times \exp (-448 / \mathrm{T})$ \\
\hline $\mathrm{ISOR}+\mathrm{HO} 2 \rightarrow 0.282 \mathrm{BiPER}+0.030 \mathrm{BiDER}+\mathrm{HO} 2$ & $2.05 \times 10^{-13} \times \exp (1300 / \mathrm{T})$ \\
\hline $\mathrm{ISOR}+\mathrm{C} 2 \mathrm{O} 3 \rightarrow 0.026 \mathrm{BiMT}+0.219 \mathrm{MACR}+\mathrm{C} 2 \mathrm{O} 3$ & $8.40 \times 10^{-14} \times \exp (221 / \mathrm{T})$ \\
\hline $\mathrm{ISOR}+\mathrm{MeO} 2 \rightarrow 0.026 \mathrm{BiMT}+0.219 \mathrm{MACR}+\mathrm{MeO} 2$ & $3.40 \times 10^{-14} \times \exp (221 / \mathrm{T})$ \\
\hline $\mathrm{ISOR}+\mathrm{NO} \rightarrow 0.418 \mathrm{MACR}+0.046 \mathrm{ISON}+\mathrm{NO}$ & $2.43 \times 10^{-12} \times \exp (360 / \mathrm{T})$ \\
\hline $\mathrm{ISOR}+\mathrm{NO} 3 \rightarrow 0.438 \mathrm{MACR}+\mathrm{NO} 3$ & $1.20 \times 10^{-12}$ \\
\hline $\mathrm{ISON}+\mathrm{OH} \rightarrow \mathrm{OH}$ & $1.30 \times 10^{-11}$ \\
\hline $\mathrm{ISON}+\mathrm{NO} 3 \rightarrow 0.074 \mathrm{BiNIT} 3+\mathrm{NO} 3$ & $6.61 \times 10^{-13}$ \\
\hline $\mathrm{MACR}+\mathrm{NO} \rightarrow \mathrm{NO}$ & $2.54 \times 10^{-12} \times \exp (360 / \mathrm{T})$ \\
\hline $\mathrm{MACR}+\mathrm{HO} 2 \rightarrow \mathrm{HO} 2$ & $1.82 \times 10^{-13} \times \exp (1300 / \mathrm{T})$ \\
\hline $\mathrm{MACR}+\mathrm{MeO} 2 \rightarrow \mathrm{MeO} 2$ & $3.40 \times 10^{-14} \times \exp (221 / \mathrm{T})$ \\
\hline $\mathrm{MACR}+\mathrm{NO} 2 \rightarrow \mathrm{MPAN}+\mathrm{NO} 2$ & $2.80 \times 10^{-12} \times \exp (181 / \mathrm{T})$ \\
\hline $\mathrm{MPAN} \rightarrow \mathrm{MACR}$ & $1.60 \times 10^{16} \times \exp (-13486 / \mathrm{T})$ \\
\hline $\mathrm{MPAN}+\mathrm{OH} \rightarrow 0.067 \mathrm{BiMGA}+0.047 \mathrm{BiNGA}+\mathrm{OH}$ & $3.20 \times 10^{-11}$ \\
\hline $\mathrm{MPAN}+\mathrm{NO} 3 \rightarrow 0.067 \mathrm{BiMGA}+0.047 \mathrm{BiNGA}+\mathrm{NO} 3$ & $3.20 \times 10^{-11}$ \\
\hline BiPER $+\mathrm{h} v \rightarrow$ Degradation products & $\mathrm{k}=50 \times$ kinetic of photolysis of $\mathrm{H}_{2} \mathrm{O}_{2}$ \\
\hline $\mathrm{API}+\mathrm{OH} \rightarrow 0.30 \mathrm{BiA} 0 \mathrm{D}+0.17 \mathrm{BiA} 1 \mathrm{D}+0.10 \mathrm{BiA} 2 \mathrm{D}+\mathrm{OH}$ & $1.21 \times 10^{-11} \times \exp (440 / \mathrm{T})$ \\
\hline $\mathrm{API}+\mathrm{O} 3 \rightarrow 0.18 \mathrm{BiA} 0 \mathrm{D}+0.16 \mathrm{BiA} 1 \mathrm{D}+0.05 \mathrm{BiA} 2 \mathrm{D}+\mathrm{O} 3$ & $5.00 \times 10^{-16} \times \exp (-530 / \mathrm{T})$ \\
\hline $\mathrm{API}+\mathrm{NO} 3 \rightarrow 0.70 \mathrm{BiA0D}+0.10 \mathrm{BiNIT}+\mathrm{NO} 3$ & $1.19 \times 10^{-12} \times \exp (-490 / \mathrm{T})$ \\
\hline $\mathrm{BPI}+\mathrm{OH} \rightarrow 0.07 \mathrm{BiA} 0 \mathrm{D}+0.08 \mathrm{BiA} 1 \mathrm{D}+0.06 \mathrm{BiA} 2 \mathrm{D}+\mathrm{OH}$ & $2.38 \times 10^{-11} \times \exp (357 / \mathrm{T})$ \\
\hline $\mathrm{BPI}+\mathrm{O} 3 \rightarrow 0.09 \mathrm{BiA} 0 \mathrm{D}+0.13 \mathrm{BiA} 1 \mathrm{D}+0.04 \mathrm{BiA} 2 \mathrm{D}+\mathrm{O} 3$ & $1.50 \times 10^{-17}$ \\
\hline $\mathrm{BPI}+\mathrm{NO} 3 \rightarrow 0.02 \mathrm{BiA} 0 \mathrm{D}+0.63 \mathrm{BiNIT}+\mathrm{NO} 3$ & $2.51 \times 10^{-12}$ \\
\hline $\mathrm{LIM}+\mathrm{OH} \rightarrow 0.35 \mathrm{BiA} 0 \mathrm{D}+0.20 \mathrm{BiA} 1 \mathrm{D}+0.0035 \mathrm{BiA} 2 \mathrm{D}+$ & \\
\hline $\mathrm{OH}$ & $4.20 \times 10^{-11} \times \exp (401 / \mathrm{T})$ \\
\hline $\mathrm{LIM}+\mathrm{O} 3 \rightarrow 0.09 \mathrm{BiA} 0 \mathrm{D}+0.10 \mathrm{BiA} 1 \mathrm{D}+\mathrm{O} 3$ & $2.95 \times 10^{-15} \times \exp (783 / \mathrm{T})$ \\
\hline $\mathrm{LIM}+\mathrm{NO} 3 \rightarrow 0.69 \mathrm{BiA} 0 \mathrm{D}+0.27 \mathrm{BiNIT}+\mathrm{NO} 3$ & $1.22 \times 10^{-11}$ \\
\hline $\mathrm{HUM}+\mathrm{OH} \rightarrow 0.74 \mathrm{BiBmP}+0.26 \mathrm{BiBlP}+\mathrm{OH}$ & $2.93 \times 10^{-10}$ \\
\hline $\mathrm{TOL}+\mathrm{OH} \rightarrow \ldots+0.25 \mathrm{TOLP}$ & $1.80 \times 10^{-12} \times \exp (355 / \mathrm{T})$ \\
\hline $\mathrm{TOLP}+\mathrm{HO} 2 \rightarrow 0.78 \mathrm{AnClP}+\mathrm{HO} 2$ & $3.75 \times 10^{-13} \times \exp (980 / \mathrm{T})$ \\
\hline $\mathrm{TOLP}+\mathrm{C} 2 \mathrm{O} 3 \rightarrow 0.78 \mathrm{AnClP}+\mathrm{C} 2 \mathrm{O} 3$ & $7.40 \times 10^{-13} \times \exp (765 / \mathrm{T})$ \\
\hline $\mathrm{TOLP}+\mathrm{MeO} 2 \rightarrow 0.78 \mathrm{AnClP}+\mathrm{MeO} 2$ & $3.56 \times 10^{-14} \times \exp (708 / \mathrm{T})$ \\
\hline $\mathrm{TOLP}+\mathrm{NO} \rightarrow 0.097 \mathrm{AnBlP}+0.748 \mathrm{AnBmP}+\mathrm{NO}$ & $2.70 \times 10^{-12} \times \exp (360 / \mathrm{T})$ \\
\hline $\mathrm{TOLP}+\mathrm{NO} 3 \rightarrow 0.097 \mathrm{AnBlP}+0.748 \mathrm{AnBmP}+\mathrm{NO} 3$ & $1.2 \times 10^{-12}$ \\
\hline $\mathrm{XYL}+\mathrm{OH} \rightarrow \ldots+0.274 \mathrm{XYLP}$ & $1.70 \times 10^{-11} \times \exp (116 / \mathrm{T})$ \\
\hline $\mathrm{XYLP}+\mathrm{HO} 2 \rightarrow 0.71 \mathrm{AnClP}+\mathrm{HO} 2$ & $3.75 \times 10^{-13} \times \exp (980 / \mathrm{T})$ \\
\hline $\mathrm{XYLP}+\mathrm{C} 2 \mathrm{O} 3 \rightarrow 0.71 \mathrm{AnClP}+\mathrm{C} 2 \mathrm{O} 3$ & $7.40 \times 10^{-13} \times \exp (765 / \mathrm{T})$ \\
\hline $\mathrm{XYLP}+\mathrm{MeO} 2 \rightarrow 0.71 \mathrm{AnClP}+\mathrm{MeO} 2$ & $3.56 \times 10^{-14} \times \exp (708 / \mathrm{T})$ \\
\hline $\mathrm{XYLP}+\mathrm{NO} \rightarrow 0.063 \mathrm{AnBlP}+0.424 \mathrm{AnBmP}+\mathrm{NO}$ & $2.70 \times 10^{-12} \times \exp (360 / \mathrm{T})$ \\
\hline $\mathrm{XYLP}+\mathrm{NO} 3 \rightarrow 0.063 \mathrm{AnBlP}+0.424 \mathrm{AnBmP}+\mathrm{NO} 3$ & $1.2 \times 10^{-12}$ \\
\hline $\mathrm{POAlP}+\mathrm{OH} \rightarrow \mathrm{SOAlP}$ & $2.0 \times 10^{-11}$ \\
\hline $\mathrm{POAmP}+\mathrm{OH} \rightarrow \mathrm{SOAmP}$ & $2.0 \times 10^{-11}$ \\
\hline $\mathrm{POAhP}+\mathrm{OH} \rightarrow \mathrm{SOAhP}$ & $2.0 \times 10^{-11}$ \\
\hline $\mathrm{BOAlP}+\mathrm{OH} \rightarrow$ BSOAlP & $2.0 \times 10^{-11}$ \\
\hline $\mathrm{BOAmP}+\mathrm{OH} \rightarrow \mathrm{BSOAmP}$ & $2.0 \times 10^{-11}$ \\
\hline $\mathrm{BOAhP}+\mathrm{OH} \rightarrow \mathrm{BSOAhP}$ & $2.0 \times 10^{-11}$ \\
\hline
\end{tabular}




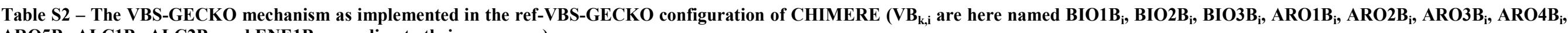
$A R O 5 B_{i}, A_{1 L C 1}, A L C 2 B_{i}$, and ENE1 $B_{i}$ according to their precursor).

\begin{tabular}{|c|c|c|}
\hline Reactions & $\begin{array}{l}\text { Kinetic rate parameters } \\
\left(\mathrm{s}^{-1} \text { or molecule }{ }^{-1} \cdot \mathrm{cm}^{3} \cdot \mathrm{s}^{-1}\right)\end{array}$ & Notes \\
\hline $\mathrm{ISOP}+\mathrm{OH} \rightarrow \mathrm{ISOR}+\mathrm{OH}$ & $2.54 \times 10^{-11} \times \exp (408 / \mathrm{T})$ & \\
\hline $\mathrm{ISOP}+\mathrm{NO} 3 \rightarrow I S O N+\mathrm{NO} 3$ & $3.03 \times 10^{-12} \times \exp (-448 / \mathrm{T})$ & \\
\hline $\mathrm{ISOR}+\mathrm{HO} 2 \rightarrow 0.282 \mathrm{BiPER}+0.030 \mathrm{BiDER}+\mathrm{HO} 2$ & $2.05 \times 10^{-13} \times \exp (1300 / \mathrm{T})$ & \\
\hline $\mathrm{ISOR}+\mathrm{C2O} 3 \rightarrow 0.026 \mathrm{BiMT}+0.219 \mathrm{MACR}+\mathrm{C} 2 \mathrm{O} 3$ & $8.40 \times 10^{-14} \times \exp (221 / \mathrm{T})$ & \\
\hline $\mathrm{ISOR}+\mathrm{MeO} 2 \rightarrow 0.026 \mathrm{BiMT}+0.219 \mathrm{MACR}+\mathrm{MeO} 2$ & $3.40 \times 10^{-14} \times \exp (221 / \mathrm{T})$ & \\
\hline$I S O R+N O \rightarrow 0.418 M A C R+0.046 I S O N+N O$ & $2.43 \times 10^{-12} \times \exp (360 / \mathrm{T})$ & \\
\hline$I S O R+N O 3 \rightarrow 0.438 M A C R+N O 3$ & $1.20 \times 10^{-12}$ & \\
\hline $\mathrm{ISON}+\mathrm{OH} \rightarrow \mathrm{OH}$ & $1.30 \times 10^{-11}$ & \\
\hline $\mathrm{ISON}+\mathrm{NO} 3 \rightarrow 0.074 \mathrm{BiNIT3}+\mathrm{NO} 3$ & $6.61 \times 10^{-13}$ & \\
\hline$M A C R+N O \rightarrow N O$ & $2.54 \times 10^{-12} \times \exp (360 / \mathrm{T})$ & \\
\hline $\mathrm{MACR}+\mathrm{HO} 2 \rightarrow \mathrm{HO} 2$ & $1.82 \times 10^{-13} \times \exp (1300 / \mathrm{T})$ & \\
\hline $\mathrm{MACR}+\mathrm{MeO} 2 \rightarrow \mathrm{MeO} 2$ & $3.40 \times 10^{-14} \times \exp (221 / \mathrm{T})$ & \\
\hline $\mathrm{MACR}+\mathrm{NO} 2 \rightarrow \mathrm{MPAN}+\mathrm{NO} 2$ & $2.80 \times 10^{-12} \times \exp (181 / \mathrm{T})$ & \\
\hline$M P A N \rightarrow M A C R$ & $1.60 \times 10^{16} \times \exp (-13486 / \mathrm{T})$ & \\
\hline$M P A N+O H \rightarrow 0.067 B i M G A+0.047 B i N G A+O H$ & $3.20 \times 10^{-11}$ & \\
\hline$M P A N+N O 3 \rightarrow 0.067 B i M G A+0.047 B i N G A+N O 3$ & $3.20 \times 10^{-11}$ & \\
\hline BiPER $+h v \rightarrow$ Degradation products & $\mathrm{k}=50 \times$ kinetic of photolysis of $\mathrm{H}_{2} \mathrm{O}_{2}$ & \\
\hline $\mathrm{APINEN}+\mathrm{OH} \rightarrow \mathrm{a}_{1} \mathrm{BIO} 1 \mathrm{~B} 1+\mathrm{a}_{2} \mathrm{BIO} 1 \mathrm{~B} 2+\mathrm{a}_{3} \mathrm{BIO} 1 \mathrm{~B} 3+\mathrm{a}_{4} \mathrm{BIO} 1 \mathrm{~B} 4+\mathrm{a}_{5} \mathrm{BIO} 1 \mathrm{~B} 5+\mathrm{a}_{6} \mathrm{BIO} 1 \mathrm{~B} 6+\mathrm{a}_{7} \mathrm{BIO} 1 \mathrm{~B} 7+\mathrm{OH}$ & $1.21 \times 10^{-11} \times \exp (440 / \mathrm{T})$ & * \\
\hline APINEN + O3 $\rightarrow \mathrm{a}_{1}$ BIO1B1 $+\mathrm{a}_{2}$ BIO1B2 $+\mathrm{a}_{3} \mathrm{BIO} 1 \mathrm{~B} 3+\mathrm{a}_{4} \mathrm{BIO} 1 \mathrm{~B} 4+\mathrm{a}_{5} \mathrm{BIO1B} 5+\mathrm{a}_{6}$ BIO1B6 $+\mathrm{a}_{7} \mathrm{BIO} 1 \mathrm{~B} 7+\mathrm{O} 3$ & $5.00 \times 10^{-16} \times \exp (-530 / \mathrm{T})$ & * \\
\hline $\mathrm{APINEN}+\mathrm{NO} 3 \rightarrow \mathrm{a}_{1} \mathrm{BIO} 1 \mathrm{~B} 1+\mathrm{a}_{2} \mathrm{BIO} 1 \mathrm{~B} 2+\mathrm{a}_{3} \mathrm{BIO} 1 \mathrm{~B} 3+\mathrm{a}_{4} \mathrm{BIO} 1 \mathrm{~B} 4+\mathrm{a}_{5} \mathrm{BIO} 1 \mathrm{~B} 5+\mathrm{a}_{6} \mathrm{BIO} 1 \mathrm{~B} 6+\mathrm{a}_{7} \mathrm{BIO} 1 \mathrm{~B} 7+\mathrm{NO} 3$ & $1.19 \times 10^{-12} \times \exp (-490 / \mathrm{T})$ & $*$ \\
\hline $\mathrm{BIO} 1 \mathrm{~B}(1-6)+\mathrm{OH} \rightarrow \mathrm{a}_{1} \mathrm{BIO} 1 \mathrm{~B} 1+\mathrm{a}_{2} \mathrm{BIO} 1 \mathrm{~B} 2+\mathrm{a}_{3} \mathrm{BIO} 1 \mathrm{~B} 3+\mathrm{a}_{4} \mathrm{BIO} 1 \mathrm{~B} 4+\mathrm{a}_{5} \mathrm{BIO} 1 \mathrm{~B} 5+\mathrm{a}_{6} \mathrm{BIO} 1 \mathrm{~B} 6+\mathrm{a}_{7} \mathrm{BIO} 1 \mathrm{~B} 7+\mathrm{OH}$ & $4.0 \times 10^{-11}$ & * \\
\hline $\mathrm{BIO} 1 \mathrm{~B}(1-6)+\mathrm{h} v \rightarrow$ Degradation products & $\mathrm{k}=11.5 \times$ kinetic of photolysis of acetone & \\
\hline $\mathrm{BPINEN}+\mathrm{OH} \rightarrow \mathrm{a}_{1} \mathrm{BIO} 2 \mathrm{~B} 1+\mathrm{a}_{2} \mathrm{BIO} 2 \mathrm{~B} 2+\mathrm{a}_{3} \mathrm{BIO} 2 \mathrm{~B} 3+\mathrm{a}_{4} \mathrm{BIO} 2 \mathrm{~B} 4+\mathrm{a}_{5} \mathrm{BIO} 2 \mathrm{~B} 5+\mathrm{a}_{6} \mathrm{BIO} 2 \mathrm{~B} 6+\mathrm{a}_{7} \mathrm{BIO} 2 \mathrm{~B} 7+\mathrm{OH}$ & $2.38 \times 10^{-11} \times \exp (357 / \mathrm{T})$ & * \\
\hline $\mathrm{BPINEN}+\mathrm{O} 3 \rightarrow \mathrm{a}_{1} \mathrm{BIO} 2 \mathrm{~B} 1+\mathrm{a}_{2} \mathrm{BIO} 2 \mathrm{~B} 2+\mathrm{a}_{3} \mathrm{BIO} 2 \mathrm{~B} 3+\mathrm{a}_{4} \mathrm{BIO} 2 \mathrm{~B} 4+\mathrm{a}_{5} \mathrm{BIO} 2 \mathrm{~B} 5+\mathrm{a}_{6} \mathrm{BIO} 2 \mathrm{~B} 6+\mathrm{a}_{7} \mathrm{BIO} 2 \mathrm{~B} 7+\mathrm{O} 3$ & $1.50 \times 10^{-17}$ & $*$ \\
\hline $\mathrm{BPINEN}+\mathrm{NO} 3 \rightarrow \mathrm{a}_{1} \mathrm{BIO} 2 \mathrm{~B} 1+\mathrm{a}_{2} \mathrm{BIO} 2 \mathrm{~B} 2+\mathrm{a}_{3} \mathrm{BIO} 2 \mathrm{~B} 3+\mathrm{a}_{4} \mathrm{BIO} 2 \mathrm{~B} 4+\mathrm{a}_{5} \mathrm{BIO} 2 \mathrm{~B} 5+\mathrm{a}_{6} \mathrm{BIO} 2 \mathrm{~B} 6+\mathrm{a}_{7} \mathrm{BIO} 2 \mathrm{~B} 7+\mathrm{NO} 3$ & $2.51 \times 10^{-12}$ & * \\
\hline $\mathrm{BIO} 2 \mathrm{~B}(1-6)+\mathrm{OH} \rightarrow \mathrm{a}_{1} \mathrm{BIO} 2 \mathrm{~B} 1+\mathrm{a}_{2} \mathrm{BIO} 2 \mathrm{~B} 2+\mathrm{a}_{3} \mathrm{BIO} 2 \mathrm{~B} 3+\mathrm{a}_{4} \mathrm{BIO} 2 \mathrm{~B} 4+\mathrm{a}_{5} \mathrm{BIO} 2 \mathrm{~B} 5+\mathrm{a}_{6} \mathrm{BIO} 2 \mathrm{~B} 6+\mathrm{a}_{7} \mathrm{BIO} 2 \mathrm{~B} 7+\mathrm{OH}$ & $4.0 \times 10^{-11}$ & * \\
\hline $\mathrm{BIO} 2 \mathrm{~B}(1-6)+\mathrm{h} v \rightarrow$ Degradation products & $\mathrm{k}=23.5 \times$ kinetic of photolysis of acetone & \\
\hline $\mathrm{LIMONE}+\mathrm{OH} \rightarrow \mathrm{a}_{1} \mathrm{BIO} 3 \mathrm{~B} 1+\mathrm{a}_{2} \mathrm{BIO} 3 \mathrm{~B} 2+\mathrm{a}_{3} \mathrm{BIO} 3 \mathrm{~B} 3+\mathrm{a}_{4} \mathrm{BIO} 3 \mathrm{~B} 4+\mathrm{a}_{5} \mathrm{BIO} 3 \mathrm{~B} 5+\mathrm{a}_{6} \mathrm{BIO} 3 \mathrm{~B} 6+\mathrm{a}_{7} \mathrm{BIO} 3 \mathrm{~B} 7+\mathrm{OH}$ & $4.20 \times 10^{-11} \times \exp (401 / \mathrm{T})$ & * \\
\hline $\mathrm{LIMONE}+\mathrm{O} 3 \rightarrow \mathrm{a}_{1} \mathrm{BIO} 3 \mathrm{~B} 1+\mathrm{a}_{2} \mathrm{BIO} 3 \mathrm{~B} 2+\mathrm{a}_{3} \mathrm{BIO} 3 \mathrm{~B} 3+\mathrm{a}_{4} \mathrm{BIO} 3 \mathrm{~B} 4+\mathrm{a}_{5} \mathrm{BIO} 3 \mathrm{~B} 5+\mathrm{a}_{6} \mathrm{BIO} 3 \mathrm{~B} 6+\mathrm{a}_{7} \mathrm{BIO} 3 \mathrm{~B} 7+\mathrm{O} 3$ & $2.95 \times 10^{-15} \times \exp (783 / \mathrm{T})$ & * \\
\hline $\mathrm{LIMONE}+\mathrm{NO} 3 \rightarrow \mathrm{a}_{1} \mathrm{BIO} 3 \mathrm{~B} 1+\mathrm{a}_{2} \mathrm{BIO} 3 \mathrm{~B} 2+\mathrm{a}_{3} \mathrm{BIO} 3 \mathrm{~B} 3+\mathrm{a}_{4} \mathrm{BIO} 3 \mathrm{~B} 4+\mathrm{a}_{5} \mathrm{BIO} 3 \mathrm{~B} 5+\mathrm{a}_{6} \mathrm{BIO} 3 \mathrm{~B} 6+\mathrm{a}_{7} \mathrm{BIO} 3 \mathrm{~B} 7+\mathrm{NO} 3$ & $1.22 \times 10^{-11}$ & $*$ \\
\hline $\mathrm{BIO} 3 \mathrm{~B}(1-6)+\mathrm{OH} \rightarrow \mathrm{a}_{1} \mathrm{BIO} 3 \mathrm{~B} 1+\mathrm{a}_{2} \mathrm{BIO} 3 \mathrm{~B} 2+\mathrm{a}_{3} \mathrm{BIO} 3 \mathrm{~B} 3+\mathrm{a}_{4} \mathrm{BIO} 3 \mathrm{~B} 4+\mathrm{a}_{5} \mathrm{BIO} 3 \mathrm{~B} 5+\mathrm{a}_{6} \mathrm{BIO} 3 \mathrm{~B} 6+\mathrm{a}_{7} \mathrm{BIO} 3 \mathrm{~B} 7+\mathrm{OH}$ & $4.0 \times 10^{-11}$ & * \\
\hline $\mathrm{BIO} 3 \mathrm{~B}(1-6)+\mathrm{h} v \rightarrow$ Degradation products & $\mathrm{k}=23.3 \times$ kinetic of photolysis of acetone & \\
\hline $\mathrm{OCIMEN}+\mathrm{OH} \rightarrow \mathrm{a}_{1} \mathrm{BIO} 3 \mathrm{~B} 1+\mathrm{a}_{2} \mathrm{BIO} 3 \mathrm{~B} 2+\mathrm{a}_{3} \mathrm{BIO} 3 \mathrm{~B} 3+\mathrm{a}_{4} \mathrm{BIO} 3 \mathrm{~B} 4+\mathrm{a}_{5} \mathrm{BIO} 3 \mathrm{~B} 5+\mathrm{a}_{6} \mathrm{BIO} 3 \mathrm{~B} 6+\mathrm{a}_{7} \mathrm{BIO} 3 \mathrm{~B} 7+\mathrm{OH}$ & $5.10 \times 10^{-8} / \mathrm{T}$ & * \\
\hline $\mathrm{OCIMEN}+\mathrm{O} 3 \rightarrow \mathrm{a}_{1} \mathrm{BIO} 3 \mathrm{~B} 1+\mathrm{a}_{2} \mathrm{BIO} 3 \mathrm{~B} 2+\mathrm{a}_{3} \mathrm{BIO} 3 \mathrm{~B} 3+\mathrm{a}_{4} \mathrm{BIO} 3 \mathrm{~B} 4+\mathrm{a}_{5} \mathrm{BIO} 3 \mathrm{~B} 5+\mathrm{a}_{6} \mathrm{BIO} 3 \mathrm{~B} 6+\mathrm{a}_{7} \mathrm{BIO} 3 \mathrm{~B} 7+\mathrm{O} 3$ & $7.50 \times 10^{-14} / \mathrm{T}$ & * \\
\hline $\mathrm{OCIMEN}+\mathrm{NO} 3 \rightarrow \mathrm{a}_{1} \mathrm{BIO} 3 \mathrm{~B} 1+\mathrm{a}_{2} \mathrm{BIO} 3 \mathrm{~B} 2+\mathrm{a}_{3} \mathrm{BIO} 3 \mathrm{~B} 3+\mathrm{a}_{4} \mathrm{BIO} 3 \mathrm{~B} 4+\mathrm{a}_{5} \mathrm{BIO} 3 \mathrm{~B} 5+\mathrm{a}_{6} \mathrm{BIO} 3 \mathrm{~B} 6+\mathrm{a}_{7} \mathrm{BIO} 3 \mathrm{~B} 7+\mathrm{NO} 3$ & $4.30 \times 10^{-9} / \mathrm{T}$ & * \\
\hline $\mathrm{HUM}+\mathrm{OH} \rightarrow 0.74 \mathrm{BiBmP}+0.26 \mathrm{BiBlP}+\mathrm{OH}$ & $2.93 \times 10^{-10}$ & \\
\hline$\overline{\mathrm{BENZEN}}+\mathrm{OH} \rightarrow \mathrm{a}_{1}$ ARO1B1 $+\mathrm{a}_{2}$ ARO1B2 $+\mathrm{a}_{3}$ ARO1B3 $+\mathrm{a}_{4}$ ARO1B4 $+\mathrm{a}_{5}$ ARO1B5 $+\mathrm{a}_{6}$ ARO1B6 $+\mathrm{a}_{7}$ ARO1B7 + OH & $1.216 \times 10^{-12}$ & * \\
\hline $\mathrm{ARO} 1 \mathrm{~B}(1-6)+\mathrm{OH} \rightarrow \mathrm{a}_{1} \mathrm{ARO} 1 \mathrm{~B} 1+\mathrm{a}_{2} \mathrm{ARO} 1 \mathrm{~B} 2+\mathrm{a}_{3} \mathrm{ARO} 1 \mathrm{~B} 3+\mathrm{a}_{4} \mathrm{ARO} 1 \mathrm{~B} 4+\mathrm{a}_{5} \mathrm{ARO} 1 \mathrm{~B} 5+\mathrm{a}_{6} \mathrm{ARO} 1 \mathrm{~B} 6+\mathrm{a}_{7} \mathrm{ARO} 1 \mathrm{~B} 7+\mathrm{OH}$ & $4.0 \times 10^{-11}$ & * \\
\hline $\operatorname{ARO} 1 \mathrm{~B}(1-6)+\mathrm{h} v \rightarrow$ Degradation products & $\mathrm{k}=1.50 \times$ kinetic of photolysis of acetone & \\
\hline TOLUEN + OH $\rightarrow a_{1}$ ARO2B1 $+a_{2}$ ARO2B2 $+a_{3}$ ARO2B3 $+a_{4}$ ARO2B $4+a_{5}$ ARO2B $5+a_{6}$ ARO2B6 $+a_{7}$ ARO2B7 + OH & $5.639 \times 10^{-12}$ & * \\
\hline $\mathrm{ARO} 2 \mathrm{~B}(1-6)+\mathrm{OH} \rightarrow \mathrm{a}_{1} \mathrm{ARO} 2 \mathrm{~B} 1+\mathrm{a}_{2} \mathrm{ARO} 2 \mathrm{~B} 2+\mathrm{a}_{3} \mathrm{ARO} 2 \mathrm{~B} 3+\mathrm{a}_{4} \mathrm{ARO} 2 \mathrm{~B} 4+\mathrm{a}_{5} \mathrm{ARO} 2 \mathrm{~B} 5+\mathrm{a}_{6} \mathrm{ARO} 2 \mathrm{~B} 6+\mathrm{a}_{7} \mathrm{ARO} 2 \mathrm{~B} 7+\mathrm{OH}$ & $4.0 \times 10^{-11}$ & * \\
\hline $\mathrm{ARO} 2 \mathrm{~B}(1-6)+\mathrm{h} v \rightarrow$ Degradation products & $\mathrm{k}=19.9 \times$ kinetic of photolysis of acetone & \\
\hline
\end{tabular}


$\mathrm{ARO} 3 \mathrm{~B}(1-6)+\mathrm{h} v \rightarrow$ Degradation products

MXYLEN + OH $\rightarrow a_{1}$ ARO4B1 $+a_{2}$ ARO4B2 $+a_{3}$ ARO4B3 $+a_{4}$ ARO4B4 + $a_{5}$ ARO4B5 $+a_{6}$ ARO4B6 + $a_{7}$ ARO4B7 + OH

$\mathrm{k}=2.30 \times$ kinetic of photolysis of acetone $2.305 \times 10^{-11}$

$\mathrm{ARO} 4 \mathrm{~B}(1-6)+\mathrm{OH} \rightarrow \mathrm{a}_{1}$ ARO4B1 $+\mathrm{a}_{2}$ ARO4B2 $+\mathrm{a}_{3} \mathrm{ARO} 4 \mathrm{~B} 3+\mathrm{a}_{4}$ ARO4B4 $+\mathrm{a}_{5}$ ARO4B5 $+\mathrm{a}_{6} \mathrm{ARO} 4 \mathrm{~B} 6+\mathrm{a}_{7} \mathrm{ARO} 4 \mathrm{~B} 7+\mathrm{OH}$ ARO4B $(1-6)+\mathrm{h} v \rightarrow$ Degradation products

$\mathrm{PXYLEN}+\mathrm{OH} \rightarrow \mathrm{a}_{1}$ ARO5B1 $+\mathrm{a}_{2}$ ARO5B2 $+\mathrm{a}_{3}$ ARO5B3 $+\mathrm{a}_{4}$ ARO5B4 $+\mathrm{a}_{5}$ ARO5B $5+\mathrm{a}_{6}$ ARO5B6 $+\mathrm{a}_{7}$ ARO5B7 + OH $\mathrm{ARO}$ B $(1-6)+\mathrm{OH} \rightarrow \mathrm{a}_{1}$ ARO5B1 $+\mathrm{a}_{2}$ ARO5B2 $+\mathrm{a}_{3}$ ARO5B3 $+\mathrm{a}_{4}$ ARO5B4 $+\mathrm{a}_{5}$ ARO5B5 + $\mathrm{a}_{6}$ ARO5B6 $+\mathrm{a}_{7} \mathrm{ARO}$ B $7+$ OH $\mathrm{ARO} \mathrm{B}(1-6)+\mathrm{h} v \rightarrow$ Degradation products

$\mathrm{ALC10}+\mathrm{OH} \rightarrow \mathrm{a}_{1}$ ALC1B1 $+\mathrm{a}_{2}$ ALC1B2 $+\mathrm{a}_{3}$ ALC1B3 $+\mathrm{a}_{4}$ ALC1B4 $+\mathrm{a}_{5}$ ALC1B5 $+\mathrm{a}_{6}$ ALC1B6 $+\mathrm{a}_{7}$ ALC1B7 + OH

$\mathrm{ALC1B}(1-6)+\mathrm{OH} \rightarrow \mathrm{a}_{1} \mathrm{ALC} 1 \mathrm{~B} 1+\mathrm{a}_{2}$ ALC1B2 $+\mathrm{a}_{3}$ ALC1B3 $+\mathrm{a}_{4}$ ALC1B4 $+\mathrm{a}_{5}$ ALC1B5 $+\mathrm{a}_{6}$ ALC1B6 $+\mathrm{a}_{7}$ ALC1B7 + OH

$\mathrm{ALC1B}(1-6)+\mathrm{h} v \rightarrow$ Degradation products

$\mathrm{ALC1} 4+\mathrm{OH} \rightarrow \mathrm{a}_{1} \mathrm{ALC} 2 \mathrm{~B} 1+\mathrm{a}_{2} \mathrm{ALC} 2 \mathrm{~B} 2+\mathrm{a}_{3} \mathrm{ALC} 2 \mathrm{~B} 3+\mathrm{a}_{4} \mathrm{ALC} 2 \mathrm{~B} 4+\mathrm{a}_{5} \mathrm{ALC} 2 \mathrm{~B} 5+\mathrm{a}_{6} \mathrm{ALC} 2 \mathrm{~B} 6+\mathrm{a}_{7} \mathrm{ALC} 2 \mathrm{~B} 7+\mathrm{OH}$

$\mathrm{ALC} 2 \mathrm{~B}(1-6)+\mathrm{OH} \rightarrow \mathrm{a}_{1} \mathrm{ALC} 2 \mathrm{~B} 1+\mathrm{a}_{2} \mathrm{ALC} 2 \mathrm{~B} 2+\mathrm{a}_{3} \mathrm{ALC} 2 \mathrm{~B} 3+\mathrm{a}_{4} \mathrm{ALC} 2 \mathrm{~B} 4+\mathrm{a}_{5} \mathrm{ALC} 2 \mathrm{~B} 5+\mathrm{a}_{6} \mathrm{ALC} 2 \mathrm{~B} 6+\mathrm{a}_{7} \mathrm{ALC} 2 \mathrm{~B} 7+\mathrm{OH}$

$\mathrm{ALC} 2 \mathrm{~B}(1-6)+\mathrm{h} v \rightarrow$ Degradation products

$\mathrm{ENE} 10+\mathrm{OH} \rightarrow \mathrm{a}_{1}$ ENE1B1 $+\mathrm{a}_{2}$ ENE1B2 $+\mathrm{a}_{3}$ ENE1B3 $+\mathrm{a}_{4}$ ENE1B4 $+\mathrm{a}_{5}$ ENE1B5 $+\mathrm{a}_{6}$ ENE1B6 $+\mathrm{a}_{7}$ ENE1B7 + OH

$\mathrm{ENE} 10+\mathrm{O} 3 \rightarrow \mathrm{a}_{1}$ ENE1B1 $+\mathrm{a}_{2}$ ENE1B2 $+\mathrm{a}_{3}$ ENE1B3 $+\mathrm{a}_{4}$ ENE1B4 $+\mathrm{a}_{5}$ ENE1B5 $+\mathrm{a}_{6}$ ENE1B6 $+\mathrm{a}_{7}$ ENE1B7 + O3

ENE10 + NO3 $\rightarrow a_{1}$ ENE1B1 $+a_{2}$ ENE1B2 $+a_{3}$ ENE1B3 $+a_{4}$ ENE1B4 $+a_{5}$ ENE1B5 $+a_{6}$ ENE1B6 $+a_{7}$ ENE1B7 + NO3

ENE1B $(1-6)+$ OH $\rightarrow a_{1}$ ENE1B1 $+a_{2}$ ENE1B2 $+a_{3}$ ENE1B3 $+a_{4}$ ENE1B4 $+a_{5}$ ENE1B5 $+a_{6}$ ENE1B6 $+a_{7}$ ENE1B7 + OH

ENE1B $(1-6)+\mathrm{h} v \rightarrow$ Degradation products

$P O A l P+O H \rightarrow S O A l P$

$\mathrm{POAmP}+\mathrm{OH} \rightarrow \mathrm{SOAmP}$

$\mathrm{POAhP}+\mathrm{OH} \rightarrow \mathrm{SOAhP}$

BOAlP $+O H \rightarrow B S O A l P$

$4.0 \times 10^{-11}$

$\mathrm{k}=2.59 \times$ kinetic of photolysis of acetone

$1.431 \times 10^{-1}$

$\mathrm{k}=16.4 \times$ kinetic of photolysis of acetone $1.099 \times 10^{-11}$ $1.099 \times 10^{-11}$
$4.0 \times 10^{-11}$

$B O A m P+O H \rightarrow B S O A m P$
$B O A h P+O H \rightarrow B S O A h P$

kinetic of photolysis of acetone

$1.678 \times 10^{-1}$ $4.0 \times 10^{-11}$ $4.402 \times 10^{-11}$ $4.402 \times 10^{-11}$
$9.290 \times 10^{-18}$

$0.265 \times 10^{-13}$ $4.0 \times 10^{-11}$

$\mathrm{k}=17.6 \times$ kinetic of photolysis of acetone

$B O A h P+O H \rightarrow B S O A h P$ $2.0 \times 10^{-11}$

$2.0 \times 10^{-11}$

$2.0 \times 10^{-11}$

$2.0 \times 10^{-11}$

$2.0 \times 10^{-11}$

Reactions in italic are taken from the $\mathrm{H}^{2} \mathrm{O}$ mechanism

* the $\mathrm{VB}_{\mathrm{k}, \mathrm{i}}$ are formed according to RRR and reactive species dependent $\mathrm{a}_{\mathrm{x}}$ stoichiometric coefficients (cf Lannuque et al., 2018) 


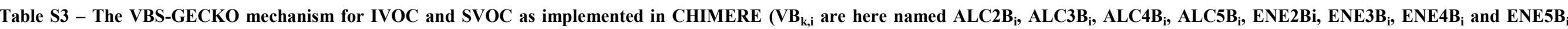
according to their precursor).

\begin{tabular}{|c|c|c|}
\hline Reactions & $\begin{array}{l}\text { Kinetic rate parameters } \\
\left(\mathrm{s}^{-1} \text { or molecule }{ }^{-1} \cdot \mathrm{cm}^{3} \cdot \mathrm{s}^{-1}\right)\end{array}$ & Notes \\
\hline$A L C 14+O H \rightarrow a_{1} A L C 2 B 1+a_{2} A L C 2 B 2+a_{3} A L C 2 B 3+a_{4} A L C 2 B 4+a_{5} A L C 2 B 5+a_{6} A L C 2 B 6+a_{7} A L C 2 B 7+O H$ & $1.678 \times 10^{-11}$ & * \\
\hline$A L C 2 B(1-6)+O H \rightarrow a_{1} A L C 2 B 1+a_{2} A L C 2 B 2+a_{3} A L C 2 B 3+a_{4} A L C 2 B 4+a_{5} A L C 2 B 5+a_{6} A L C 2 B 6+a_{7} A L C 2 B 7+O H$ & $4.0 \times 10^{-11}$ & * \\
\hline$A L C 2 B(1-6)+h v \rightarrow$ Degradation products & $\mathrm{k}=19.4 \times$ kinetic of photolysis of aceton & \\
\hline $\mathrm{ALC} 18+\mathrm{OH} \rightarrow \mathrm{a}_{1} \mathrm{ALC} 3 \mathrm{~B} 1+\mathrm{a}_{2}$ ALC3B2 $+\mathrm{a}_{3} \mathrm{ALC} 3 \mathrm{~B} 3+\mathrm{a}_{4}$ ALC3B4 $+\mathrm{a}_{5}$ ALC3B5 $+\mathrm{a}_{6}$ ALC3B6 $+\mathrm{a}_{7}$ ALC3B7 + OH & $2.244 \times 10^{-11}$ & * \\
\hline $\mathrm{ALC} 3 \mathrm{~Bq} 1-6)+\mathrm{OH} \rightarrow \mathrm{a}_{1} \mathrm{ALC} 3 \mathrm{~B} 1+\mathrm{a}_{2} \mathrm{ALC} 3 \mathrm{~B} 2+\mathrm{a}_{3} \mathrm{ALC} 3 \mathrm{~B} 3+\mathrm{a}_{4} \mathrm{ALC} 3 \mathrm{~B} 4+\mathrm{a}_{5} \mathrm{ALC} 3 \mathrm{~B} 5+\mathrm{a}_{6} \mathrm{ALC} 3 \mathrm{~B} 6+\mathrm{a}_{7} \mathrm{ALC} 3 \mathrm{~B} 7+\mathrm{OH}$ & $4.0 \times 10^{-11}$ & * \\
\hline $\mathrm{ALC} 3 \mathrm{~B}(1-6)+\mathrm{h} v \rightarrow$ Degradation products & $\mathrm{k}=6.39 \times$ kinetic of photolysis of aceton & \\
\hline $\mathrm{ALC} 22+\mathrm{OH} \rightarrow \mathrm{a}_{1}$ ALC4B1 $+\mathrm{a}_{2}$ ALC4B2 $+\mathrm{a}_{3}$ ALC4B3 $+\mathrm{a}_{4}$ ALC4B4 + $\mathrm{a}_{5}$ ALC4B5 $+\mathrm{a}_{6}$ ALC4B6 $+\mathrm{a}_{7}$ ALC4B7 + OH & $2.811 \times 10^{-11}$ & * \\
\hline $\mathrm{ALC} 4 \mathrm{~B}(1-6)+\mathrm{OH} \rightarrow \mathrm{a}_{1} \mathrm{ALC} 4 \mathrm{~B} 1+\mathrm{a}_{2} \mathrm{ALC} 4 \mathrm{~B} 2+\mathrm{a}_{3} \mathrm{ALC} 4 \mathrm{~B} 3+\mathrm{a}_{4} \mathrm{ALC} 4 \mathrm{~B} 4+\mathrm{a}_{5} \mathrm{ALC} 4 \mathrm{~B} 5+\mathrm{a}_{6} \mathrm{ALC} 4 \mathrm{~B} 6+\mathrm{a}_{7} \mathrm{ALC} 4 \mathrm{~B} 7+\mathrm{OH}$ & $4.0 \times 10^{-11}$ & * \\
\hline $\operatorname{ALC} 4 \mathrm{~B}(1-6)+\mathrm{h} v \rightarrow$ Degradation products & $\mathrm{k}=6.25 \times$ kinetic of photolysis of aceton & \\
\hline $\mathrm{ALC} 26+\mathrm{OH} \rightarrow \mathrm{a}_{1}$ ALC5B1 $+\mathrm{a}_{2}$ ALC5B2 $+\mathrm{a}_{3}$ ALC5B3 $+\mathrm{a}_{4}$ ALC5B $4+\mathrm{a}_{5}$ ALC5B5 + $\mathrm{a}_{6}$ ALC5B6+ & $3.377 \times 10^{-11}$ & * \\
\hline ALC5B $(1-6)+O H \rightarrow a_{1}$ ALC5B1 $+a_{2}$ ALC5B2 $+a_{3}$ ALC5B3 $+a_{4}$ ALC5B4 $+a_{5}$ ALC5B5 $+a_{6}$ ALC5 & $4.0 \times 10^{-11}$ & * \\
\hline $\operatorname{ALC} 5 \mathrm{~B}(1-6)+\mathrm{h} v \rightarrow$ Degradation products & $\mathrm{k}=0.00 \times$ kinetic of photolysis of aceton & \\
\hline ENE14 + OH $\rightarrow a_{1}$ ENE2B1 $+a_{2}$ ENE2B2 $+a_{3}$ ENE2B3 $+a_{4}$ ENE2B4 $+a_{5}$ ENE2B5 $+a_{6}$ ENE2B6 $+a_{7}$ ENE2B7 + OH & $4.970 \times 10^{-11}$ & * \\
\hline $\mathrm{ENE} 14+\mathrm{O} 3 \rightarrow \mathrm{a}_{1}$ ENE2B1 $+\mathrm{a}_{2}$ ENE2B2 $+\mathrm{a}_{3}$ ENE2B3 $+\mathrm{a}_{4}$ ENE2B4 $+\mathrm{a}_{5}$ ENE2B5 $+\mathrm{a}_{6}$ ENE2B6 $+\mathrm{a}_{7}$ ENE2B7 + O3 & $1.011 \times 10^{-17}$ & * \\
\hline $\mathrm{ENE} 14+\mathrm{NO} 3 \rightarrow{ }_{1}$ ENE2B1 $+\mathrm{a}_{2}$ ENE2B2 $+\mathrm{a}_{3}$ ENE2B3 $+\mathrm{a}_{4}$ ENE2B4 $+\mathrm{a}_{5}$ ENE2B5 $+\mathrm{a}_{6}$ ENE2B6 $+\mathrm{a}_{7}$ ENE2B7 + NO3 & $0.305 \times 10^{-13}$ & * \\
\hline ENE2B $(1-6)+\mathrm{OH} \rightarrow \mathrm{a}_{1}$ ENE2B1 $+\mathrm{a}_{2}$ ENE2B2 $+\mathrm{a}_{3}$ ENE2B3 $+\mathrm{a}_{4}$ ENE2B4 $+\mathrm{a}_{5}$ ENE2B5 $+\mathrm{a}_{6}$ ENE2B6 $+\mathrm{a}_{7}$ ENE2B7 + OH & $4.0 \times 10^{-11}$ & * \\
\hline ENE2B $(1-6)+\mathrm{h} v \rightarrow$ Degradation products & $\mathrm{k}=9.26 \times$ kinetic of photolysis of aceton & \\
\hline ENE18 + OH $\rightarrow a_{1}$ ENE3B1 $+a_{2}$ ENE3B2 $+a_{3}$ ENE3B3 $+a_{4}$ ENE3B4 $+a_{5}$ ENE3B5 $+a_{6}$ ENE3B6 $+a_{7}$ ENE3B7 + OH & $5.537 \times 10^{-11}$ & * \\
\hline $\mathrm{ENE} 18+\mathrm{O} 3 \rightarrow \mathrm{a}_{1}$ ENE3B1 $+\mathrm{a}_{2}$ ENE3B2 $+\mathrm{a}_{3}$ ENE3B3 $+\mathrm{a}_{4}$ ENE3B4 $+\mathrm{a}_{5}$ ENE3B5 $+\mathrm{a}_{6}$ ENE3B6 $+\mathrm{a}_{7}$ ENE3B7 + O3 & $1.011 \times 10^{-17}$ & * \\
\hline ENE18 + NO3 $\rightarrow a_{1}$ ENE3B1 $+a_{2}$ ENE3B2 $+a_{3}$ ENE3B3 $+a_{4}$ ENE3B4 $+a_{5}$ ENE3B5 $+a_{6}$ ENE3B6 $+a_{7}$ ENE3B7 + NO3 & $0.326 \times 10^{-13}$ & * \\
\hline ENE3B $(1-6)+\mathrm{OH} \rightarrow \mathrm{a}_{1}$ ENE3B1 $+\mathrm{a}_{2}$ ENE3B2 $+\mathrm{a}_{3}$ ENE3B3 $+\mathrm{a}_{4}$ ENE3B $4+\mathrm{a}_{5}$ ENE3B5 $+\mathrm{a}_{6}$ ENE3B6 $+\mathrm{a}_{7}$ ENE3B7 + OH & $4.0 \times 10^{-11}$ & * \\
\hline ENE3B $(1-6)+\mathrm{h} v \rightarrow$ Degradation products & $\mathrm{k}=2.74 \times$ kinetic of photolysis of aceton & \\
\hline ENE22 + OH $\rightarrow a_{1}$ ENE4B1 $+a_{2}$ ENE4B2 $+a_{3}$ ENE4B3 $+a_{4}$ ENE4B4 $+a_{5}$ ENE4B5 $+a_{6}$ ENE4B6 $+a_{7}$ ENE4B7 + OH & $6.105 \times 10^{-11}$ & * \\
\hline ENE22 + O3 $\rightarrow a_{1}$ ENE4B1 $+a_{2}$ ENE4B2 $+a_{3}$ ENE4B3 $+a_{4}$ ENE4B4 $+a_{5}$ ENE4B5 $+a_{6}$ ENE4B6 $+a_{7}$ ENE4B7 + O3 & $1.011 \times 10^{-17}$ & * \\
\hline ENE22 + NO3 $\rightarrow a_{1}$ ENE4B1 $+a_{2}$ ENE4B2 $+a_{3}$ ENE4B3 $+a_{4}$ ENE4B4 $+a_{5}$ ENE4B5 $+a_{6}$ ENE4B6 $+a_{7}$ ENE4B7 + NO3 & $0.337 \times 10^{-13}$ & * \\
\hline ENE4B $(1-6)+\mathrm{OH} \rightarrow \mathrm{a}_{1}$ ENE4B1 $+\mathrm{a}_{2}$ ENE4B2 $+\mathrm{a}_{3}$ ENE4B3 $+\mathrm{a}_{4}$ ENE4B4 $+\mathrm{a}_{5}$ ENE4B5 $+\mathrm{a}_{6}$ ENE4B6 $+\mathrm{a}_{7}$ ENE4B7 + OH & $4.0 \times 10^{-11}$ & * \\
\hline ENE4B $(1-6)+h v \rightarrow$ Degradation products & $\mathrm{k}=6.13 \times$ kinetic of photolysis of aceton & \\
\hline ENE26 + OH $\rightarrow a_{1}$ ENE5B1 $+a_{2}$ ENE5B2 $+a_{3}$ ENE5B3 $+a_{4}$ ENE5B4 $+a_{5}$ ENE5B5 $+a_{6}$ ENE5B6 $+a_{7}$ ENE5B7 + OH & $6.673 \times 10^{-11}$ & * \\
\hline ENE26 + O3 $\rightarrow a_{1}$ ENE5B1 $+a_{2}$ ENE5B2 $+a_{3}$ ENE5B3 $+a_{4}$ ENE5B4 $+a_{5}$ ENE5B5 $+a_{6}$ ENE5B6 $+a_{7}$ ENE5B7 + O3 & $1.011 \times 10^{-17}$ & * \\
\hline ENE26 + NO3 $\rightarrow a_{1}$ ENE5B1 $+a_{2}$ ENE5B2 $+a_{3}$ ENE5B3 $+a_{4}$ ENE5B4 $+a_{5}$ ENE5B5 $+a_{6}$ ENE5B6 $+a_{7}$ ENE5B7 + NO3 & $0.343 \times 10^{-13}$ & * \\
\hline ENE5B(1-6) + OH $\rightarrow a_{1}$ ENE5B1 $+a_{2}$ ENE5B2 $+a_{3}$ ENE5B3 $+a_{4}$ ENE5B4 $+a_{5}$ ENE5B5 $+a_{6}$ ENE5B6 $+a_{7}$ ENE5B7 + OH & $4.0 \times 10^{-11}$ & * \\
\hline & & \\
\hline
\end{tabular}

$\mathrm{ENE} 5 \mathrm{~B}(1-6)+\mathrm{h} v \rightarrow$ Degradation products

Reactions in italic are already in the ref-VBS-GECKO configuration of CHIMERE

$\mathrm{k}=0.00 \times$ kinetic of photolysis of aceton

* the $\mathrm{VB}_{\mathrm{k}, \mathrm{i}}$ are formed according to RRR and reactive species dependent $\mathrm{a}_{\mathrm{x}}$ stoichiometric coefficients (cf Lannuque et al., 2018) 


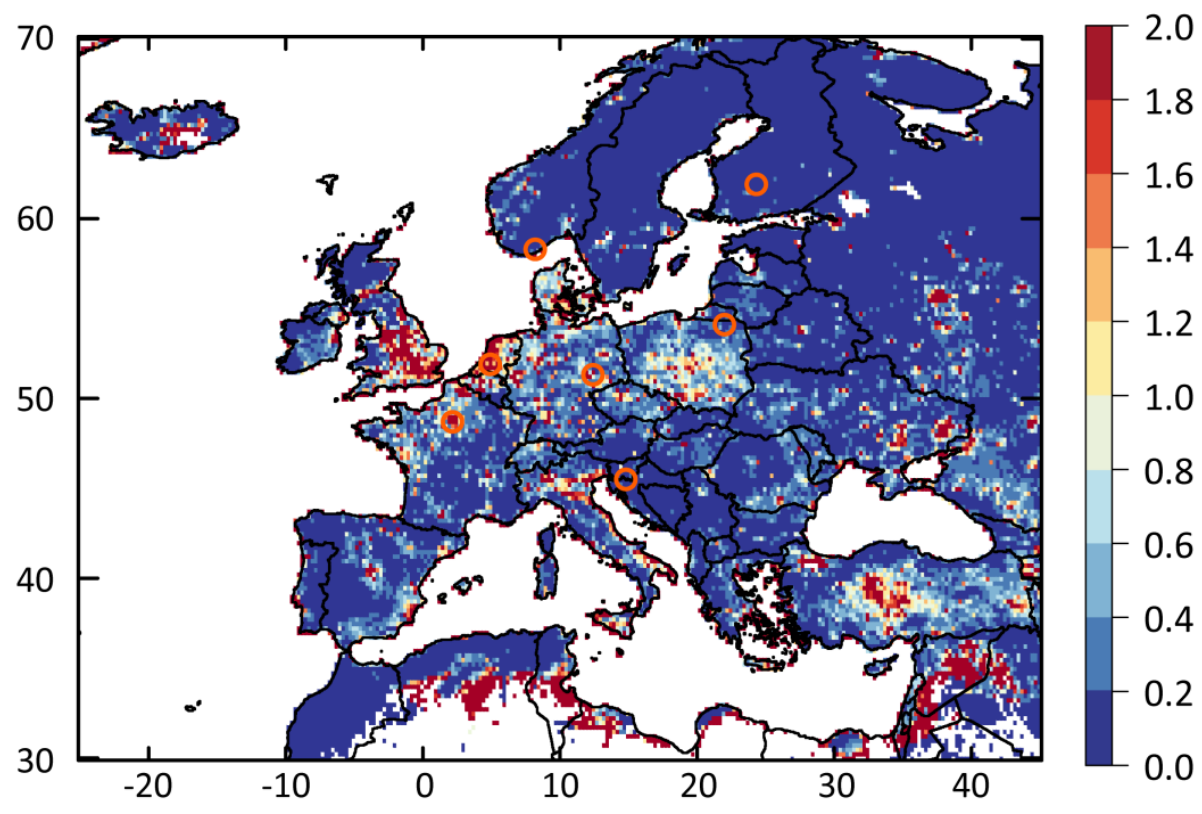

Figure S1 - Mean simulated emission ${ }_{\text {toluene }} /$ emission $_{\alpha-p i n e n e}$ ratio for July-August 2013 over Europe. Circles represent stations used 5 for time series comparison. White pixels represent limit values of the ratio (typically when at least one of the emission fluxes is null). 

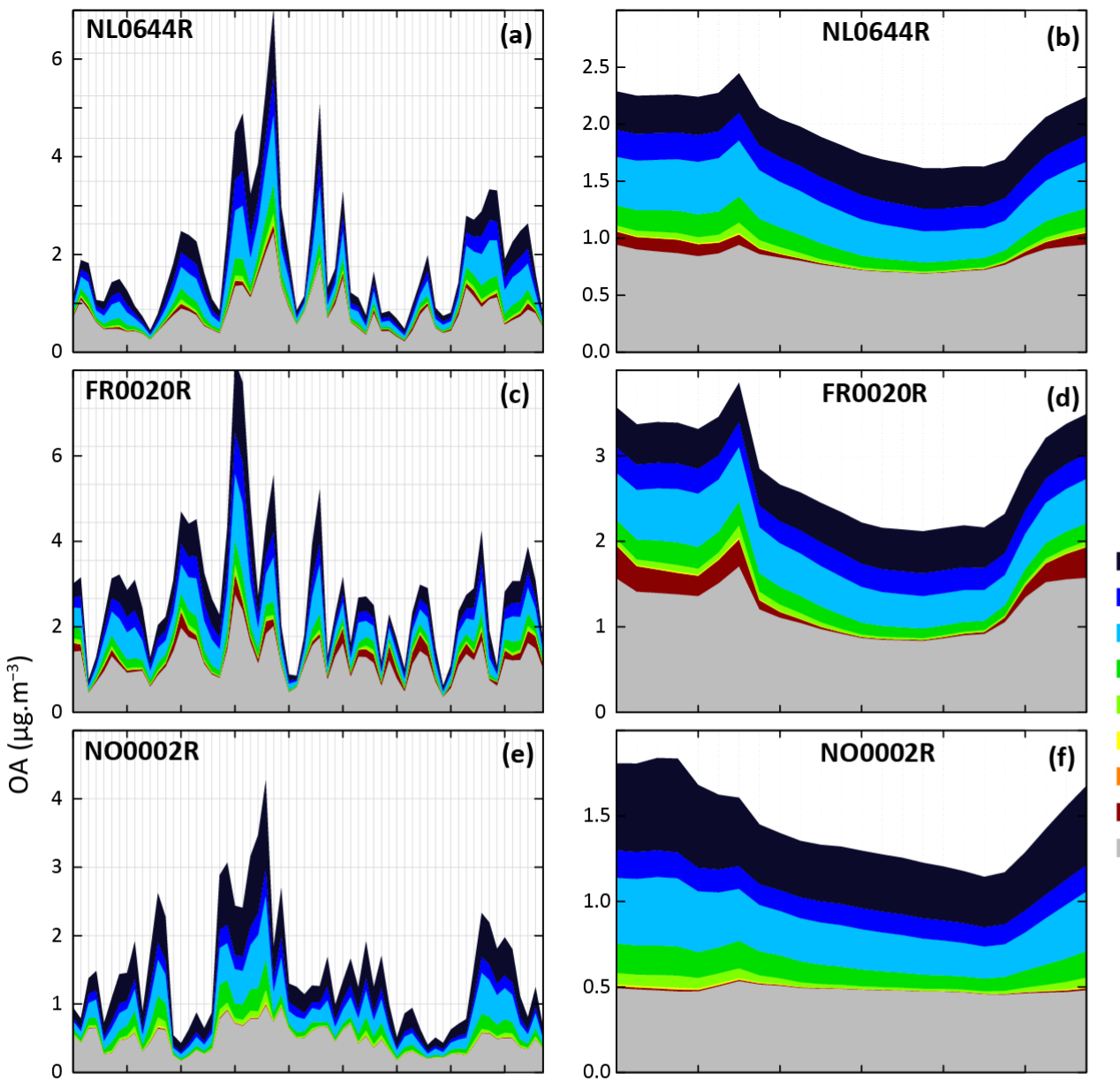

VB3

VB2

- VB1

traffic SVOC

others
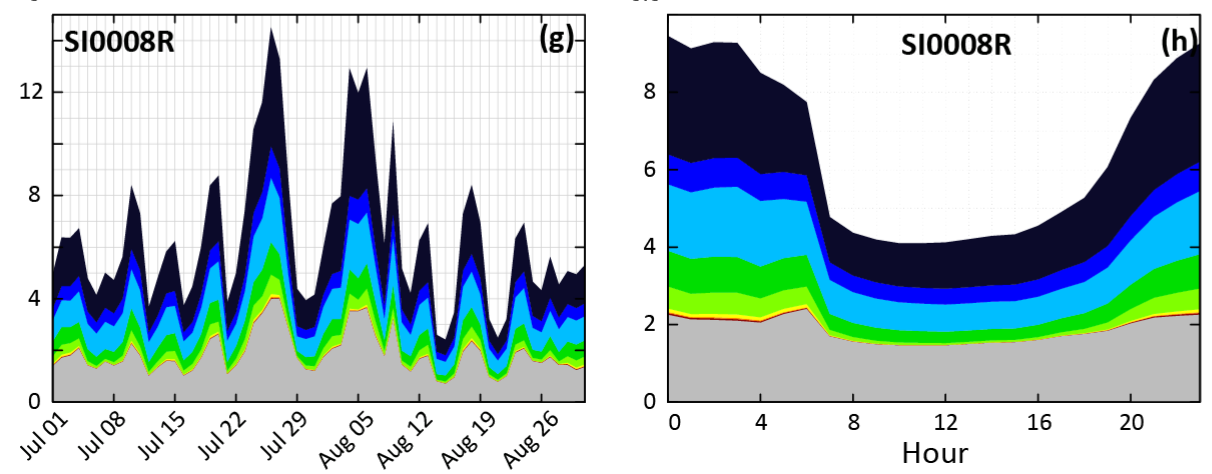

Figure S2 - Evolution of simulated OA concentrations and distribution function of volatility bins with the IVOC $30 \mathrm{voc}$ model configuration (from the less volatile one VB7, to the more volatile VB1). Panels $b, d, f$ and $h$ present mean daily profiles. Results are shown at two stations influenced by anthropogenic sources in Netherland (NL0644R, a and b) and in France (FR0020R, $c$ and 5 d) and at two stations influenced by biogenic sources in Norway (NL0002R, e and f) and Slovenia (SI0008R, g and h). "Traffic SVOC" includes $\mathrm{C}_{14}$ to $\mathrm{C}_{26}$ emitted VBS-GECKO alkanes and alkenes and "others" includes all species from $\mathrm{H}^{2} \mathrm{O}$ mechanism. 


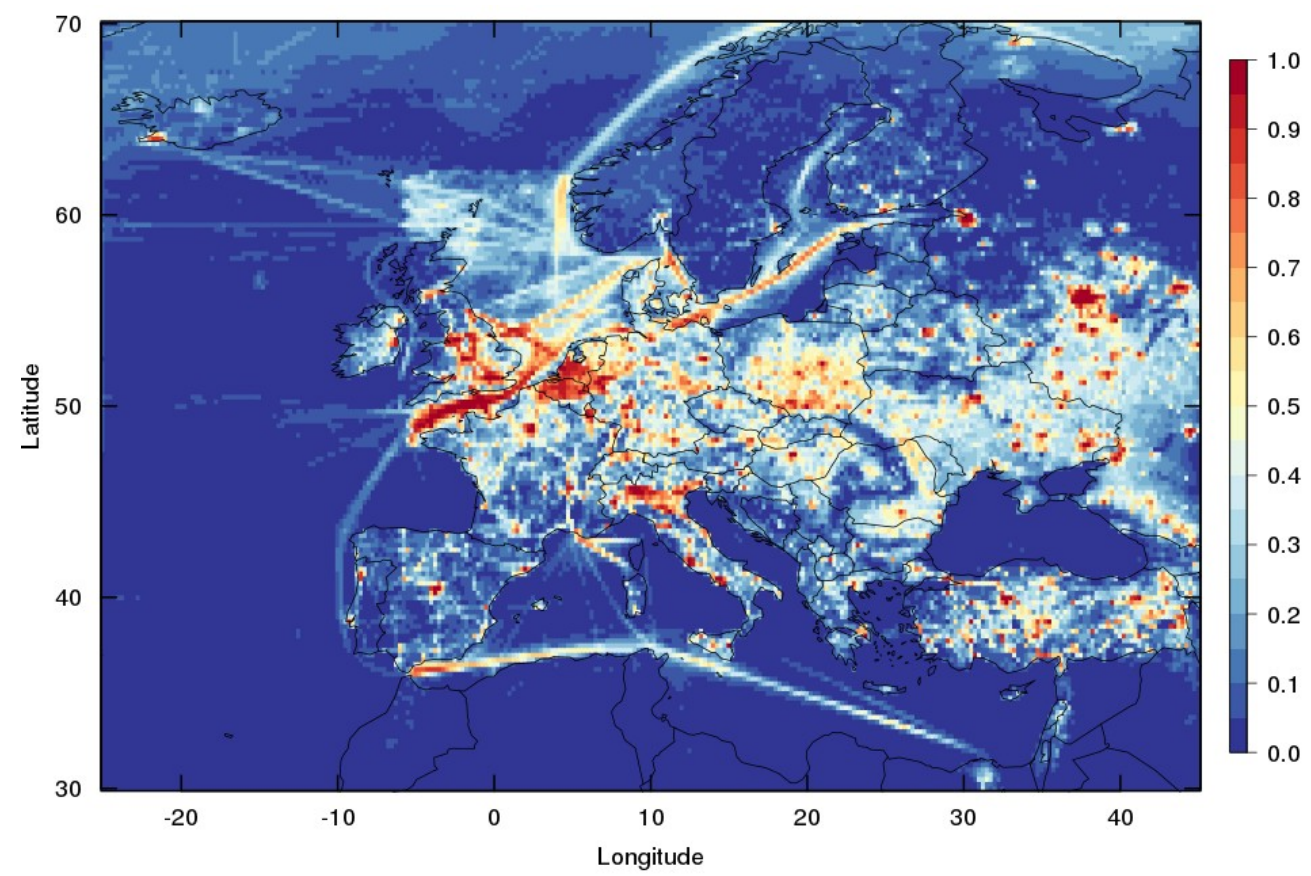

Figure S3 - Mean RRR over Europe during the two-month period for the ref-VBS-GECKO model configuration. 